\title{
Identification of a circadian gene signature that predicts overall survival in lung adenocarcinoma
}

\author{
Xinliang Gao ${ }^{1}$, Mingbo Tang ${ }^{1}$, Suyan Tian ${ }^{2}$, Jialin ${ }^{1}{ }^{1}$, Wei Liu ${ }^{\text {Corresp. } 1}$ \\ ${ }^{1}$ Department of Thoracic Surgery, The First Hospital of Jilin University, Changchun, Jilin Province, China \\ 2 Division of Clinical Research, The First Hospital of Jilin University, Changchun, Jilin Province, China \\ Corresponding Author: Wei Liu \\ Email address: I_w01@jlu.edu.cn
}

Background. Lung adenocarcinoma (LUAD) is one of the most common subtypes of lung cancer which is the leading cause of death in cancer patients. Circadian clock disruption has been listed as a likely carcinogen. However, whether the expression of circadian genes affects overall survival (OS) in LUAD patients remains unknown. In this article, we identified a circadian gene signature to predict overall survival in LUAD . Methods. RNA sequencing (HTSeq-FPKM) data and clinical characteristics were obtained for a cohort of LUAD patients from The Cancer Genome Atlas (TCGA). A multigene signature based on differentially expressed circadian clock-related genes was generated for the prediction of OS using Least Absolute Shrinkage and Selection Operator (LASSO)-penalized Cox regression analysis, and externally validated using the GSE72094 dataset from the GEO database. Results. Five differentially expressed genes (DEGs) were identified to be significantly associated with OS using univariate Cox proportional regression analysis $(P<$ 0.05). Patients classified as high risk based on these 5 DEGs had significantly lower OS than those classified as low risk in both the TGCA cohort and GSE72094 dataset (P < 0.001). Multivariate Cox regression analysis revealed that the five-gene-signature based risk score was an independent predictor of OS (hazard ratio $>1, P<0.001$ ). Receiver operating characteristic (ROC) curves confirmed its prognostic value. Gene set enrichment analysis (GSEA) showed that Kyoto Encyclopedia of Genes and Genomes (KEGG) pathways related to cell proliferation, gene damage repair, proteasomes, and immune and autoimmune diseases were significantly enriched. Conclusion. A novel circadian gene signature for OS in LUAD was found to be predictive in both the derivation and validation cohorts. Targeting circadian genes is a potential therapeutic option in LUAD. 


\section{Identification of a Circadian Gene Signature that}

2 Predicts Overall Survival in Lung Adenocarcinoma

5 Xinliang Gao ${ }^{1}$, Mingbo Tang ${ }^{1}$, Suyan Tian ${ }^{2}$, Jialin $\mathrm{Li}^{1}$, Wei Liu ${ }^{1}$

6

$7{ }^{1}$ Department of Thoracic Surgery, The First Hospital of Jilin University, Changchun, Jilin

8 Province, PR China

$9{ }^{2}$ Division of Clinical Research, The First Hospital of Jilin University, Changchun, Jilin

10 Province, PR China

12 Corresponding Author:

13 Wei Liu ${ }^{1}$

1471 Xinmin Streat, Changchun, Jilin Province, 130021, PR China

15 Email address: 1_w01@jlu.edu.cn

16 


\section{Abstract}

19 Background. Lung adenocarcinoma (LUAD) is one of the most common subtypes of lung cancer which is the leading cause of death in cancer patients. Circadian clock disruption has been listed as a likely carcinogen. However, whether the expression of circadian genes affects overall survival (OS) in LUAD patients remains unknown. In this article, we identified a circadian gene signature to predict overall survival in LUAD.

Methods. RNA sequencing (HTSeq-FPKM) data and clinical characteristics were obtained for a cohort of LUAD patients from The Cancer Genome Atlas (TCGA). A multigene signature based on differentially expressed circadian clock-related genes was generated for the prediction of OS using Least Absolute Shrinkage and Selection Operator (LASSO)-penalized Cox regression analysis, and externally validated using the GSE72094 dataset from the GEO database.

Results. Five differentially expressed genes (DEGs) were identified to be significantly associated with OS using univariate Cox proportional regression analysis $(\mathrm{P}<0.05)$. Patients classified as high risk based on these 5 DEGs had significantly lower OS than those classified as low risk in both the TGCA cohort and GSE72094 dataset $(\mathrm{P}<0.001)$. Multivariate Cox regression analysis revealed that the five-gene-signature based risk score was an independent predictor of OS (hazard ratio $>1, \mathrm{P}<0.001)$. Receiver operating characteristic $(\mathrm{ROC})$ curves confirmed its prognostic value. Gene set enrichment analysis (GSEA) showed that Kyoto Encyclopedia of Genes and Genomes (KEGG) pathways related to cell proliferation, gene damage repair, proteasomes, and immune and autoimmune diseases were significantly enriched.

Conclusion. A novel circadian gene signature for OS in LUAD was found to be predictive in both the derivation and validation cohorts. Targeting circadian genes is a potential therapeutic option in LUAD.

\section{Introduction} survival rate is only 19\% (Siegel et al. 2019). In 2019, there were 228,150 new diagnoses of 
cancers of the lung and bronchus in the United States. Primary lung cancer is divided into two main types: small-cell lung carcinoma and non-small cell lung carcinoma (NSCLC). The latter is further classified into different subtypes according to the histological origin, such as lung adenocarcinoma (LUAD), squamous cell carcinoma, or large cell carcinoma. Among these, LUAD is the most prevalent subtype, with an increasing incident in recent years (Cheng et al. 2016). The prognosis of LUAD is improving due to advances in molecular targeted treatment and immunotherapy (Hirsch et al. 2016; Peters et al. 2019). However, accurate prognosis prediction models for LUAD are still lacking.

The circadian clock is a molecular time-keeping system that is evolutionarily conserved. It is vital for the maintenance of physiologic homeostasis and normal function in all organisms. It coordinates a variety of biological processes and behaviors (Fu \& Kettner 2013; Panda et al. 2002). In the suprachiasmatic nucleus ( $\mathrm{SCN})$ of the hypothalamus, a central clock maintains the daily rhythms in the body by neural and humoral communication with peripheral clocks located in peripheral tissues and regulates bodily functions such as sleep/wake cycles and the secretion of many hormones. Disruption of the circadian clock has been listed as a likely carcinogen by the World Health Organization based on both population and laboratory-based findings (Lunn et al. 2017; Straif et al. 2007), which raised the interest in research on the relationship between circadian genes and tumor development. Some circadian genes have been demonstrated to control the occurrence and development of NSCLC (Qiu et al. 2019). However, the association between circadian genes and prognosis in patients with LUAD remains to be elucidated.

The present study aims to explore the prognostic role of circadian genes in patients with LUAD using The Cancer Genome Atlas (TCGA) data obtained from the NCI Genomic Data Commons, which includes the clinical characteristics and mRNA expression profiles of tumor and tumoradjacent normal tissues. A prognostic multigene signature will be established using differentially expressed circadian clock genes and then validated with the GSE72094 dataset extracted from the Gene Expression Omnibus (GEO) database. Underlying molecular mechanisms were investigated by performing a Gene set enrichment analysis (GSEA) with Kyoto Encyclopedia of Genes and Genomes (KEGG) pathways. 


\section{Materials \& Methods}

\section{Data collection}

The clinical characteristics and RNA sequencing data (HTSeq-FPKM) of 515 patients with LUAD were retrieved from the NCI Genomic Data Commons (https://portal.gdc.cancer.gov/repository). These 515 patients provided 535 samples from LUAD tumor tissue and 59 samples from adjacent normal tissue. Among the patients, 500 had complete RNA sequencing data and 469 had both complete sequencing data and complete clinical information.

The differential expression of the following 14 core genes of the circadian clock according to previous literature was analyzed: Period 1 (PER1), PER2, PER3, Cryptochrome Circadian Regulator 1 (CRY1), CRY2, Circadian Locomotor Output Cycles Kaput (CLOCK), Aryl Hydrocarbon Receptor Nuclear Translocator Like (ARNTL), Timeless Circadian Regulator (TIMELESS), Neuronal PAS Domain Protein 2 (NPAS2), Nuclear Receptor Subfamily 1 Group D Member 1 (NR1D1), NR1D2, Basic Helix-Loop-Helix Family Member E40 (BHLHE40), BHLHE41, and RAR-Related Orphan Receptor A (RORA) (Chen et al. 2020; Cox \& Takahashi 2019; Mocellin et al. 2018; Shafi \& Knudsen 2019; Yu et al. 2019). The validation dataset was obtained from the GSE72094 dataset in the GEO database (https://www.ncbi.nlm.nih.gov/geo/) and included microarray and clinical data for 443 LUAD tumor samples (Schabath et al. 2016). The normalized count data were downloaded. The data cut-off date was September 10, 2020. Patients with no follow-up data or information on the expression of circadian genes were excluded. The TCGA and GEO databases are public data repositories and therefore, ethical approval for this study was not required. This study followed the polices and guidelines for data access and publication specified by the TCGA and GEO databases.

\section{Prognostic validity of the gene signature}

Differentially expressed genes (DEGs) involved in the circadian clock were analyzed in the tumor and tumor-adjacent normal tissues of LUAD patients from the TCGA cohort using the "limma" package in $\mathrm{R}$ (false discovery rate $(\mathrm{FDR})<0.05)$. Univariate Cox regression analysis was used to identify circadian genes related to overall survival (OS). A gene signature for the prediction of OS was constructed with the DEGs for the circadian clock using Least Absolute Shrinkage and Selection Operator (LASSO)-penalized Cox regression analysis and the "glmnet" package in R. 
102

103

104

105

106

107

108

109

110

111

112

113

114

115

116

117

118

119

120

121

122

123

124

125

126

127

128

129

130

DEGs served as independent variables, and OS as the response variable.

A risk score based on the expression of identified candidate genes was calculated for each patient according to the following formula: score $=$ sum (normalized gene expression level $\times$ regression coefficient). Patients were classified as either high- or low-risk using the median score as the cutoff value. The survival analysis of different risk groups was determined with the "survminer" $\mathrm{R}$ package. In order to validate the performance of the signature, we used the principal components analysis (PCA) and t-distributed stochastic neighbor embedding (t-SNE) to analyze dimensionality reduction. The "prcomp" function in the R "stats" package was used to carry out the PCA. The data distribution for high-risk and low-risk patients was also mapped using t-SNE and the "Rtsne" package in R. The predictive value of the gene signature was evaluated with time-dependent Receiver operating characteristics (ROC) curve analysis using the "timeROC" package in R. The associations between the risk score, clinical characteristics (gender, age, smoking history, and stage), and OS were assessed with univariate and multivariate Cox regression analyses.

\section{Functional enrichment analysis}

The DEGs between the high- and low-risk groups in the TCGA LUAD cohort were identified using the "limma" R package again. GSEA of these DEGs was carried out with KEGG pathways ( $\mid \log 2$ fold change $\mid \geq 1$, FDR $<0.05)$. Both a nominal P-value $<0.05$ and FDR q-value $<0.05$ were considered statistically significant.

\section{Statistical analysis}

All statistical analyses were conducted with the R software (Version 3.5.3) and SPSS software (Version 25.0). Gene expression was compared using the two-tailed Student's t-test and proportions were compared using the Chi-squared test. The Kaplan-Meier method and the logrank test were used to assess the differences in OS between high and low-risk patients. Univariate and multivariate Cox regression analyses were used to identify independent predictors of OS. P < 0.05 (two tailed) was considered statistically significant.

\section{Results}

\section{Clinical and demographic characteristics}


131 Two patient cohorts with available data on OS and the RNA expression of circadian clock genes

132 were used to create the prognostic model. The derivation cohort consisted of 500 patients with

133 LUAD and complete RNA sequencing data from the TCGA database while the validation cohort

134 consisted of 398 patients with LUAD from the GSE72094 dataset. Among these patients, 469

135 patients from TCGA and 328 patients from GSE72094 who not only had complete RNA

136 sequencing data, but also complete clinical data including OS, age, gender, smoking history, and

137 tumor stage, were included in the univariate and multivariate COX analyses. The validation cohort

138 had higher age, lower TNM stage, more smokers, and a higher median OS compared to the

139 derivation cohort. The baseline demographic and clinical characteristics of the included patients

140 are summarized in Table 1.

141 Identification of DEGs related to circadian clock in the TGCA LUAD cohort

142 In the TCGA LUAD cohort, 9/14 circadian genes were found to be differentially expressed 143 between tumor and tumor-adjacent normal tissues. Five candidate genes were identified to be 144 significantly associated with OS using univariate Cox proportional regression analysis (Figure 1A145 B). The clustering of the 5 candidate genes are shown with a heatmap in Figure $1 \mathrm{C}$.

146 Generation of a prognostic signature in the TGCA LUAD cohort

147 The 5 identified candidate genes were incorporated into a five-gene-signature based prognostic 148 model using LASSO Cox regression analysis. According to risk scores calculated using the 149 expression levels of these 5 genes, half of the patients were classified as high-risk $(n=250)$ and the other half as low-risk ( $\mathrm{n}=250)$ (Figure 2A). The chance of survival was lower and the survival time was shorter in the high-risk group than in the low-risk group (Figure 2B). PCA and t-SNE analysis showed discernible dimensions between high-risk and low-risk patients (Figure 2C-D). Kaplan-

153

154

155

156

157

158 159

Meier survival curves confirmed that OS was significantly worse in high-risk than in low-risk patients (Figure 2E, $\mathrm{P}<0.001$ ). The predictive performance of the five-gene-signature based risk score for OS was evaluated using time-dependent ROC curves. The area under the curve (AUC) values were: 1 year, 0.726; 2 years, 0.668; and 3 years, 0.657 (Figure 2F).

\section{Validation of the five-gene-signature based prognostic model}

The stringency of the model developed using the TGCA LUAD cohort was validated in the GSE72094 dataset. Risk scores were calculated for all patients based on the expression levels of 
160 the 5 identified candidate genes and patients were classified as high-risk or low-risk accordingly

161 (Figure 3A). The high-risk group had a significantly higher chance of death and lower OS time

162 (Figure 3B). PCA and t-SNE analysis showed discernible dimensions between high- and low-risk

163 patients (Figure 3C-D). Kaplan-Meier survival curves confirmed that OS was significantly worse

164 in high-risk patients (Figure 3E, $\mathrm{P}<0.001$ ). The AUC values were: 1 year, 0.621; 2 years, 0.657;

165 and 3 years, 0.642 (Figure $3 F$ ).

166 Prognostic value of the five-gene-signature based risk score

167 Univariate and multivariate Cox regression analyses were conducted to determine whether the 168 five-gene-signature based risk score was an independent predictor of OS (Table 2). The derivation 169 cohort consisted of 469 patients from the TCGA LUAD cohort; and the validation cohort consisted 170 of 328 patients from the GSE72094 dataset. Univariate regression analysis revealed that the risk 171 score was significantly associated with OS in both the TGCA LUAD cohort and the GSE72094 172 dataset (TGCA LUAD cohort: hazard ratio $(\mathrm{HR})=3.373,95 \%$ confidence interval $(\mathrm{CI})=2.199$ 5.174, $\mathrm{P}<0.001$; GSE72094 dataset: $\mathrm{HR}=2.16395 \% \mathrm{CI}=1.393-3.358, \mathrm{P}<0.001)$. The risk

score was found to be an independent predictor of OS even after correcting for confounders in multivariate Cox regression analysis (TGCA LUAD cohort: $\mathrm{HR}=3.522,95 \% \mathrm{CI}=2.260-5.487$, $\mathrm{P}<0.001$; GSE72094 dataset: $\mathrm{HR}=2.195,95 \% \mathrm{CI}=1.411-3.415, \mathrm{P}<0.001$; Figure 4A-B).

\section{Enrichment analysis in the TGCA LUAD cohort}

Genes that were differentially expressed in the high- respective low-risk groups were subjected to GSEA for KEGG pathways (Table 3). The results showed that tumorigenesis pathways related to pyrimidine metabolism, cell cycle, proteasome, base excision repair, homologous recombination, and DNA replication were enriched (Figure 5).

\section{Discussion}

Genes of the circadian clock are often abnormally expressed in tumor tissues and may play an important role in tumorigenesis (Kelleher et al. 2014; Kettner et al. 2014). The present study identified 9 DEGs between tumor and tumor-adjacent normal tissues among the 14 circadian genes. The genes PER3, CRY2, TIMELESS, NPAS2, and RORA were found to be correlated with OS. These results suggest that circadian clock genes may affect the survival outcome in LUAD 
and that a signature based on the expression of these genes may predict OS and may be an independent prognostic factor.

The PER family is generally considered to have a tumor suppressor effect, and the mechanisms behind the tumor suppressing effects of PER1 and PER2 are clear (Gery et al. 2006; Wood et al. 2008). PER3 has been confirmed to affect the susceptibility and prognosis of lung cancer through expression changes, methylation, and single nucleotide polymorphisms (SNPs) (Chu et al. 2018; Couto et al. 2014; Liu et al. 2014). However, the exact mechanism for the PER3 inhibition of tumors is not yet clear. The study by Jun-Sub et al. showed that PER3 is required for checkpoint kinase 2 ( $C H K 2)$ activation in human cells, which highlighted its potential role in cell cycle arrest and DNA damage repair (Im et al. 2010). Previous studies have linked the circadian clock gene CRY2 to the occurrence and development of many tumors (Hasakova et al. 2018; Lesicka et al. 2018; Relles et al. 2013; Tokunaga et al. 2008). As a transcriptional suppressor, $C R Y 2$ functions as an important regulator of cell cycle, proliferation, DNA damage checkpoint control, and DNA repair (Hoffman et al. 2010). CRY2 acts as a tumor suppressor gene. It can limit tumor formation by increasing c-MYC turnover (Huber et al. 2016), or increase the elimination of premalignant and malignant cells through the activation of p53-independent apoptosis pathways (Lee \& Sancar 2011). The circadian genes NPAS2 and TIMELESS, on the other hand, are both correlated with poor OS. A recent study has shown that upregulated NPAS2 promoted the survival of hepatocellular carcinoma cells through the upregulation of cell division cycle $25 A(C D C 25 A)$ and inhibition of mitochondria-dependent intrinsic apoptosis (Yuan et al. 2017). Knockout or inhibition of TIMELESS can lead to cell cycle stagnation and subsequent apoptosis, which limits the growth of liver cancer cells (Elgohary et al. 2015). The circadian gene RORA was found to be downregulated in LUAD tissue and negatively correlated with LUAD prognosis in this study. $R O R A$ is a versatile gene. Besides the circadian clock, it is also a well-known regulator of inflammation and lipid metabolism. Moreover, recent studies have suggested that RORA may also play a role in the progression and prognosis of colon cancer and breast cancer (Lee et al. 2010). The recruitment of RORA can induce the expression of the tumor suppressor genes $F-b o x / W D$ repeat-containing protein 7 (FBXW7), Semaphorin $3 F(S E M A 3 F)$, and P21, leading to apoptosis and suppression of tumor cell proliferation (Wang et al. 2017). 
218 Results from the enrichment analysis revealed that metabolic pathways related to the substrates of 219 DNA synthesis (pyrimidine metabolism and pentose phosphate pathway) were enriched in the 220 high-risk group, as well as pathways regulating cell cycle and DNA replication. Increasing 221 evidence suggests a regulatory effect of circadian genes on cellular proliferation (Chakrabarti \& Michor 2020), and their involvement in the proliferation of a variety of tumor cells (Abreu et al. 2018; Wang et al. 2016; Yu et al. 2018). A recent study on lung cancer demonstrated that the loss of the central clock components led to increased c-MYC expression, which enhanced proliferation (Papagiannakopoulos et al. 2016). Base excision repair and homologous recombination pathways were also found to be enriched in the high-risk group, which may indicate that the circadian clock disorder affects the repair of gene damage to influence the survival of malignant tumors. Both $C R Y$ and TIMELESS are known to be involved in DNA damage repair. Tae et al. found that $C R Y_{\mathrm{s}}$ are related to the nucleotide excision repair gene XPA (Kang et al. 2011). TIMELESS can modulate CHK1 and serine/threonine-protein kinase (ATR) downstream of single-strand DNA breaks and activate $C H K 2$ via $A T M$ modulation downstream of double strand breaks (Yang et al. 2010). The proteasome pathway was enriched in the high-risk group. Recent studies have also confirmed that some ubiquitin ligases participate in the degradation of core circadian clock genes through the ubiquitin-proteasome pathway, thereby controlling the biological functions of cells, including cell senescence (Chen et al. 2018; Ullah et al. 2020). This cross-talk between circadian clock genes and the ubiquitin-proteasome pathway may be related to the prognosis of LUAD. Some immune and autoimmune disease pathways were enriched in the low-risk group. This shows that the disturbance of the circadian clock is accompanied by alterations in the function of the immune system (Aiello et al. 2020), which may be related to the occurrence, development, and prognosis of LUAD. $\mathrm{Wu}$ and his colleagues have shown that abnormal circadian genes contribute to $\mathrm{T}$ cell exhaustion and global upregulation of immune inhibitory molecules, such as programmed deathligand 1 (PD-L1) and cytotoxic T-lymphocyte antigen (CTLA)-4, which promote tumor development (Wu et al. 2019).

There are several limitations to this study. Firstly, the present study is a retrospective study with data from publicly available databases. This makes the study more prone to selection bias and it is also impossible to draw conclusions regarding cause-effect. Experimental studies should be 
247 conducted to understand the mechanisms behind the role of the circadian genes. Secondly, using 248 tumor-adjacent normal tissue as a control has the advantages of minimizing biological variation, 249 but one cannot be sure if the seemingly "normal" tissue adjacent to a tumor is truly "normal". 250 Thirdly, while there might be many other genes that are important in LUAD, we only focused on 25114 core genes of the circadian clock. It is possible that other more important genes were excluded 252 from the design.

\section{Conclusions}

255 In summary, we constructed a novel 5-gene signature with genes involved in the circadian clock 256 to predict the prognosis of LUAD. The signature could successfully separate LUAD patients with 257 a low risk of non-survival from those with a high risk in both the derivation and validation cohorts. 258 The underlying molecular mechanisms between circadian genes and tumor proliferation, DNA 259 repair, ubiquitin-proteasome pathway, and immunity in LUAD remain poorly understood. and 260 warrant further investigation. Circadian genes might be potential targets for future cancer therapy.

\section{Acknowledgements}

263 This manuscript has been edited and proofread by Medjaden Bioscience Limited. 
265

266

267

268

269

270

271

272

273

274

275

276

277

278

279

280

281

282

283

284

285

286

287

288

289

290

291

292

293

\section{References}

Abreu M, Basti A, Genov N, Mazzoccoli G, and Relógio A. 2018. The reciprocal interplay between TNF $\alpha$ and the circadian clock impacts on cell proliferation and migration in Hodgkin lymphoma cells. Sci Rep 8:11474. 10.1038/s41598-018-29847-z

Aiello I, Fedele MLM, Román F, Marpegan L, Caldart C, Chiesa JJ, Golombek DA, Finkielstein $\mathrm{CV}$, and Paladino N. 2020. Circadian disruption promotes tumor-immune microenvironment remodeling favoring tumor cell proliferation. Sci Adv 6 . 10.1126/sciadv.aaz4530

Bray F, Ferlay J, Soerjomataram I, Siegel RL, Torre LA, and Jemal A. 2018. Global cancer statistics 2018: GLOBOCAN estimates of incidence and mortality worldwide for 36 cancers in 185 countries. CA Cancer J Clin 68:394-424. 10.3322/caac.21492

Chakrabarti S, and Michor F. 2020. Circadian clock effects on cellular proliferation: Insights from theory and experiments. Curr Opin Cell Biol 67:17-26. 10.1016/j.ceb.2020.07.003

Chen J, Liu A, Lin Z, Wang B, Chai X, Chen S, Lu W, Zheng M, Cao T, Zhong M, Li R, Wu M, Lu Z, Pang W, Huang W, Xiao L, Lin D, Wang Z, Lei F, Chen X, Long W, Zheng Y, Chen Q, Zeng J, Ren D, Li J, Zhang X, and Huang Y. 2020. Downregulation of the circadian rhythm regulator HLF promotes multiple-organ distant metastases in non-small cell lung cancer through PPAR/NF-kb signaling. Cancer Lett 482:56-71. 10.1016/j.canlet.2020.04.007

Chen S, Yang J, Yang L, Zhang Y, Zhou L, Liu Q, Duan C, Mieres CA, Zhou G, and Xu G. 2018. Ubiquitin ligase TRAF2 attenuates the transcriptional activity of the core clock protein BMAL1 and affects the maximal Per1 mRNA level of the circadian clock in cells. Febs $j$ 285:2987-3001. 10.1111/febs. 14595

Cheng TY, Cramb SM, Baade PD, Youlden DR, Nwogu C, and Reid ME. 2016. The International Epidemiology of Lung Cancer: Latest Trends, Disparities, and Tumor Characteristics. $J$ Thorac Oncol 11:1653-1671. 10.1016/j.jtho.2016.05.021

Chu LW, Till C, Yang B, Tangen CM, Goodman PJ, Yu K, Zhu Y, Han S, Hoque AM, Ambrosone C, Thompson I, Leach R, and Hsing AW. 2018. Circadian genes and risk of prostate cancer 
294

295

296

297

298

in the prostate cancer prevention trial. Mol Carcinog 57:462-466. 10.1002/mc.22770

Couto P, Miranda D, Vieira R, Vilhena A, De Marco L, and Bastos-Rodrigues L. 2014. Association between CLOCK, PER3 and CCRN4L with non-small cell lung cancer in Brazilian patients. Mol Med Rep 10:435-440. 10.3892/mmr.2014.2224

Cox KH, and Takahashi JS. 2019. Circadian clock genes and the transcriptional architecture of the clock mechanism. J Mol Endocrinol 63:R93-r102. 10.1530/jme-19-0153

Elgohary N, Pellegrino R, Neumann O, Elzawahry HM, Saber MM, Zeeneldin AA, Geffers R, Ehemann V, Schemmer P, Schirmacher P, and Longerich T. 2015. Protumorigenic role of Timeless in hepatocellular carcinoma. Int J Oncol 46:597-606. 10.3892/ijo.2014.2751

Fu L, and Kettner NM. 2013. The circadian clock in cancer development and therapy. Prog Mol Biol Transl Sci 119:221-282. 10.1016/b978-0-12-396971-2.00009-9

Gery S, Komatsu N, Baldjyan L, Yu A, Koo D, and Koeffler HP. 2006. The circadian gene per1 plays an important role in cell growth and DNA damage control in human cancer cells. Mol Cell 22:375-382. 10.1016/j.molcel.2006.03.038

Hasakova K, Vician M, Reis R, Zeman M, and Herichova I. 2018. Sex-dependent correlation between survival and expression of genes related to the circadian oscillator in patients with colorectal cancer. Chronobiol Int 35:1423-1434. 10.1080/07420528.2018.1488722

Hirsch FR, Suda K, Wiens J, and Bunn PA, Jr. 2016. New and emerging targeted treatments in advanced non-small-cell lung cancer. Lancet 388:1012-1024. 10.1016/s01406736(16)31473-8

Hoffman AE, Zheng T, Ba Y, Stevens RG, Yi CH, Leaderer D, and Zhu Y. 2010. Phenotypic effects of the circadian gene Cryptochrome 2 on cancer-related pathways. BMC Cancer 10:110. 10.1186/1471-2407-10-110

Huber AL, Papp SJ, Chan AB, Henriksson E, Jordan SD, Kriebs A, Nguyen M, Wallace M, Li Z, Metallo CM, and Lamia KA. 2016. CRY2 and FBXL3 Cooperatively Degrade c-MYC. Mol Cell 64:774-789. 10.1016/j.molcel.2016.10.012

Im JS, Jung BH, Kim SE, Lee KH, and Lee JK. 2010. Per3, a circadian gene, is required for Chk2 activation in human cells. FEBS Lett 584:4731-4734. 10.1016/j.febslet.2010.11.003

Kang TH, Reardon JT, and Sancar A. 2011. Regulation of nucleotide excision repair activity by 
transcriptional and post-transcriptional control of the XPA protein. Nucleic Acids Res 39:3176-3187. 10.1093/nar/gkq1318

Kelleher FC, Rao A, and Maguire A. 2014. Circadian molecular clocks and cancer. Cancer Lett 342:9-18. 10.1016/j.canlet.2013.09.040

Kettner NM, Katchy CA, and Fu L. 2014. Circadian gene variants in cancer. Ann Med 46:208-220. $10.3109 / 07853890.2014 .914808$

Lee JH, and Sancar A. 2011. Regulation of apoptosis by the circadian clock through NF-kappaB signaling. Proc Natl Acad Sci U S A 108:12036-12041. 10.1073/pnas.1108125108

Lee JM, Kim IS, Kim H, Lee JS, Kim K, Yim HY, Jeong J, Kim JH, Kim JY, Lee H, Seo SB, Kim H, Rosenfeld MG, Kim KI, and Baek SH. 2010. RORalpha attenuates Wnt/beta-catenin signaling by PKCalpha-dependent phosphorylation in colon cancer. Mol Cell 37:183-195. 10.1016/j.molcel.2009.12.022

Lesicka M, Jabłońska E, Wieczorek E, Seroczyńska B, Siekierzycka A, Skokowski J, Kalinowski L, Wąsowicz W, and Reszka E. 2018. Altered circadian genes expression in breast cancer tissue according to the clinical characteristics. PLoS One 13:e0199622. 10.1371/journal.pone.0199622

Liu B, Xu K, Jiang Y, and Li X. 2014. Aberrant expression of Per1, Per2 and Per3 and their prognostic relevance in non-small cell lung cancer. Int J Clin Exp Pathol 7:7863-7871.

Lunn RM, Blask DE, Coogan AN, Figueiro MG, Gorman MR, Hall JE, Hansen J, Nelson RJ, Panda S, Smolensky MH, Stevens RG, Turek FW, Vermeulen R, Carreón T, Caruso CC, Lawson CC, Thayer KA, Twery MJ, Ewens AD, Garner SC, Schwingl PJ, and Boyd WA. 2017. Health consequences of electric lighting practices in the modern world: A report on the National Toxicology Program's workshop on shift work at night, artificial light at night, and circadian disruption. Sci Total Environ 607-608:1073-1084. 10.1016/j.scitotenv.2017.07.056

Mocellin S, Tropea S, Benna C, and Rossi CR. 2018. Circadian pathway genetic variation and cancer risk: evidence from genome-wide association studies. BMC Med 16:20. 10.1186/s12916-018-1010-1

Panda S, Antoch MP, Miller BH, Su AI, Schook AB, Straume M, Schultz PG, Kay SA, Takahashi 
352

353

354

355

356

357

358

359

360

361

362

363

364

365

366

367

368

369

370

371

372

373

JS, and Hogenesch JB. 2002. Coordinated transcription of key pathways in the mouse by the circadian clock. Cell 109:307-320. 10.1016/s0092-8674(02)00722-5

Papagiannakopoulos T, Bauer MR, Davidson SM, Heimann M, Subbaraj L, Bhutkar A, Bartlebaugh J, Vander Heiden MG, and Jacks T. 2016. Circadian Rhythm Disruption Promotes Lung Tumorigenesis. Cell Metab 24:324-331. 10.1016/j.cmet.2016.07.001

Peters S, Reck M, Smit EF, Mok T, and Hellmann MD. 2019. How to make the best use of immunotherapy as first-line treatment of advanced/metastatic non-small-cell lung cancer. Ann Oncol 30:884-896. 10.1093/annonc/mdz109

Qiu M, Chen YB, Jin S, Fang XF, He XX, Xiong ZF, and Yang SL. 2019. Research on circadian clock genes in non-small-cell lung carcinoma. Chronobiol Int 36:739-750. $10.1080 / 07420528.2018 .1509080$

Relles D, Sendecki J, Chipitsyna G, Hyslop T, Yeo CJ, and Arafat HA. 2013. Circadian gene expression and clinicopathologic correlates in pancreatic cancer. J Gastrointest Surg 17:443-450. 10.1007/s11605-012-2112-2

Schabath MB, Welsh EA, Fulp WJ, Chen L, Teer JK, Thompson ZJ, Engel BE, Xie M, Berglund AE, Creelan BC, Antonia SJ, Gray JE, Eschrich SA, Chen DT, Cress WD, Haura EB, and Beg AA. 2016. Differential association of STK11 and TP53 with KRAS mutationassociated gene expression, proliferation and immune surveillance in lung adenocarcinoma. Oncogene 35:3209-3216. 10.1038/onc.2015.375

Shafi AA, and Knudsen KE. 2019. Cancer and the Circadian Clock. Cancer Res 79:3806-3814. 10.1158/0008-5472.Can-19-0566

Siegel RL, Miller KD, and Jemal A. 2019. Cancer statistics, 2019. CA Cancer J Clin 69:7-34. 10.3322/caac. 21551

Straif K, Baan R, Grosse Y, Secretan B, El Ghissassi F, Bouvard V, Altieri A, Benbrahim-Tallaa L, and Cogliano V. 2007. Carcinogenicity of shift-work, painting, and fire-fighting. Lancet Oncol 8:1065-1066. 10.1016/s1470-2045(07)70373-x

Tokunaga H, Takebayashi Y, Utsunomiya H, Akahira J, Higashimoto M, Mashiko M, Ito K, Niikura H, Takenoshita S, and Yaegashi N. 2008. Clinicopathological significance of circadian rhythm-related gene expression levels in patients with epithelial ovarian cancer. 
Acta Obstet Gynecol Scand 87:1060-1070. 10.1080/00016340802348286

Ullah K, Chen S, Lu J, Wang X, Liu Q, Zhang Y, Long Y, Hu Z, and Xu G. 2020. The E3 ubiquitin ligase STUB1 attenuates cell senescence by promoting the ubiquitination and degradation of the core circadian regulator BMAL1. $J$ Biol Chem 295:4696-4708. 10.1074/jbc.RA119.011280

Wang Q, Ao Y, Yang K, Tang H, and Chen D. 2016. Circadian clock gene Per2 plays an important role in cell proliferation, apoptosis and cell cycle progression in human oral squamous cell carcinoma. Oncol Rep 35:3387-3394. 10.3892/or.2016.4724

Wang Z, Xiong F, Wang X, Qi Y, Yu H, Zhu Y, and Zhu H. 2017. Nuclear receptor retinoidrelated orphan receptor alpha promotes apoptosis but is reduced in human gastric cancer. Oncotarget 8:11105-11113. 10.18632/oncotarget.14364

Wood PA, Yang X, Taber A, Oh EY, Ansell C, Ayers SE, Al-Assaad Z, Carnevale K, Berger FG, Peña MM, and Hrushesky WJ. 2008. Period 2 mutation accelerates ApcMin/+ tumorigenesis. Mol Cancer Res 6:1786-1793. 10.1158/1541-7786.Mcr-08-0196

Wu Y, Tao B, Zhang T, Fan Y, and Mao R. 2019. Pan-Cancer Analysis Reveals Disrupted Circadian Clock Associates With T Cell Exhaustion. Front Immunol 10:2451. 10.3389/fimmu.2019.02451

Yang X, Wood PA, and Hrushesky WJ. 2010. Mammalian TIMELESS is required for ATMdependent CHK2 activation and G2/M checkpoint control. J Biol Chem 285:3030-3034. 10.1074/jbc.M109.050237

Yu CC, Chen LC, Chiou CY, Chang YJ, Lin VC, Huang CY, Lin IL, Chang TY, Lu TL, Lee CH, Huang SP, and Bao BY. 2019. Genetic variants in the circadian rhythm pathway as indicators of prostate cancer progression. Cancer Cell Int 19:87. 10.1186/s12935-0190811-4

Yu M, Li W, Wang Q, Wang Y, and Lu F. 2018. Circadian regulator NR1D2 regulates glioblastoma cell proliferation and motility. Oncogene 37:4838-4853. 10.1038/s41388018-0319-8

Yuan P, Li J, Zhou F, Huang Q, Zhang J, Guo X, Lyu Z, Zhang H, and Xing J. 2017. NPAS2 promotes cell survival of hepatocellular carcinoma by transactivating CDC25A. Cell Death 


\section{Table 1 (on next page)}

Table 1. Demographical and clinical characteristics 
1 Table 1. Demographical and clinical characteristics

\begin{tabular}{c|lll}
\hline \multicolumn{1}{l}{ NCGA LUAD } & GSE72094 & P value \\
\hline $\begin{array}{l}\text { No. of patients } \\
\text { Age (median, range) }\end{array}$ & 469 & 328 & \\
Gender (\%) & $65.1(33-88)$ & $69.7(41-89)$ & $\mathrm{P}<0.01$ \\
$\quad$ Female & $257(54.8 \%)$ & $172(52.4 \%)$ & $\mathrm{P}=0.559$ \\
$\quad$ Male & $212(45.2 \%)$ & $156(47.6 \%)$ & \\
TNM Stage & & & $\mathrm{P}=0.007$ \\
$\quad$ I & $257(54.8 \%)$ & $218(66.5 \%)$ & \\
II & $112(23.9 \%)$ & $53(16.2 \%)$ & \\
III & $75(16.0 \%)$ & $46(14.0 \%)$ & \\
IV & $25(5.3 \%)$ & $11(3.4 \%)$ & \\
Smoking history & & & \\
Non-smoker & $69(14.7 \%)$ & $30(9.1 \%)$ & \\
Smoker & $400(85.3 \%)$ & $298(90.9 \%)$ & \\
Median OS (days) & 629 & 842 & $\mathrm{P}=0.034$ \\
\hline
\end{tabular}


Table 2 (on next page)

Table 2. Risk factors affecting OS in the TCGA LUAD cohort and GSE72094. 
1 Table 2. Risk factors affecting OS in the TCGA LUAD cohort and GSE72094.

\begin{tabular}{|c|c|c|c|c|c|c|c|c|c|c|c|c|}
\hline \multirow[t]{3}{*}{ Factors } & \multicolumn{6}{|c|}{ TCGA LUAD } & \multicolumn{6}{|c|}{ GSE72094 } \\
\hline & \multicolumn{3}{|c|}{ Univariate } & \multicolumn{3}{|c|}{ Multivariate } & \multicolumn{3}{|c|}{ Univariate } & \multicolumn{3}{|c|}{ Multivariate } \\
\hline & HR & $95 \% \mathrm{CI}$ & $\mathrm{P}$ & HR & $95 \% \mathrm{CI}$ & $\mathrm{P}$ & HR & $95 \% \mathrm{CI}$ & $\mathrm{P}$ & HR & $95 \% \mathrm{CI}$ & $\mathrm{P}$ \\
\hline Age & 1.01 & $0.99-1.02$ & 0.302 & 1.02 & $1.00-1.03$ & 0.026 & 1.01 & $0.98-1.03$ & 0.533 & 1.00 & $0.98-1.02$ & 0.973 \\
\hline Gender & 1.12 & $0.83-1.52$ & 0.458 & 1.09 & $0.79-1.49$ & 0.606 & 1.99 & $1.30-3.05$ & 0.002 & 2.32 & $1.49-3.62$ & 0.000 \\
\hline Stage & 1.61 & $1.39-1.85$ & 0.000 & 1.59 & $1.37-1.84$ & 0.000 & 1.70 & $1.40-2.07$ & 0.000 & 1.86 & $1.52-2.29$ & 0.000 \\
\hline Smoking & 0.91 & $0.60-1.38$ & 0.655 & 0.81 & $0.53-1.26$ & 0.351 & 1.31 & $0.57-3.01$ & 0.523 & 0.87 & $0.37-2.03$ & 0.750 \\
\hline Risk score & 3.37 & $2.20-5.17$ & 0.000 & 3.53 & $2.26-5.49$ & 0.000 & 2.16 & $1.39-3.36$ & 0.001 & 2.20 & $1.41-3.41$ & 0.000 \\
\hline
\end{tabular}

\section{2}




\section{Table 3 (on next page)}

Table 3. GSEA of DEGs between high-risk group and low-risk group with KEGG pathways. 
1 Table 3. GSEA of DEGs between high-risk group and low-risk group with KEGG pathways.

\begin{tabular}{|c|c|c|c|}
\hline KEGG pathway & NES & NOM p-val & FDR q-val \\
\hline PYRIMIDINE_METABOLISM & 2.17 & 0.000 & 0.000 \\
\hline CELL_CYCLE & 2.11 & 0.000 & 0.003 \\
\hline SPLICEOSOME & 2.08 & 0.000 & 0.005 \\
\hline PROTEASOME & 2.05 & 0.000 & 0.006 \\
\hline BASE_EXCISION_REPAIR & 2.02 & 0.000 & 0.009 \\
\hline HOMOLOGOUS_RECOMBINATION & 1.97 & 0.000 & 0.015 \\
\hline PATHOGENIC_ESCHERICHIA_COLI_INFECTION & 1.97 & 0.000 & 0.025 \\
\hline DNA_REPLICATION & 1.91 & 0.000 & 0.025 \\
\hline GLYCOSPHINGOLIPID_BIOSYNTHESIS_LACTO_AND_NEOLACTO_SERIES & 1.89 & 0.000 & 0.030 \\
\hline PENTOSE_PHOSPHATE_PATHWAY & 1.88 & 0.006 & 0.030 \\
\hline THYROID_CANCER & 1.87 & 0.002 & 0.032 \\
\hline INTESTINAL_IMMUNE_NETWORK_FOR_IGA_PRODUCTION & -2.11 & 0.000 & 0.011 \\
\hline HEMATOPOIETIC_CELL_LINEAGE & -2.04 & 0.002 & 0.018 \\
\hline ASTHMA & -1.95 & 0.002 & 0.039 \\
\hline AUTOIMMUNE_THYROID_DISEASE & -1.95 & 0.006 & 0.030 \\
\hline CELL_ADHESION_MOLECULES_CAMS & -1.95 & 0.002 & 0.025 \\
\hline PRIMARY_BILE_ACID_BIOSYNTHESIS & -1.91 & 0.000 & 0.032 \\
\hline GLYCOSPHINGOLIPID_BIOSYNTHESIS_GANGLIO_SERIES & -1.90 & 0.004 & 0.029 \\
\hline ALLOGRAFT_REJECTION & -1.89 & 0.010 & 0.031 \\
\hline TYPE_I_DIABETES_MELLITUS & -1.88 & 0.014 & 0.031 \\
\hline
\end{tabular}




\section{Figure 1}

Figure 1. Identification of the candidate genes involved in the circadian cycle in the TCGA cohort.

(A) Venn diagram of DEGs and prognostic genes that correlate with OS in tumor and tumoradjacent normal tissue. (B) Forest plots of the 5 genes that overlap between DEGs and prognostic genes that relate to OS on univariate Cox regression analysis. (C) The MRNA heatmap of 5 candidate genes. (D-H) The expression of 5 candidate genes in tumor and normal tissue. 
A

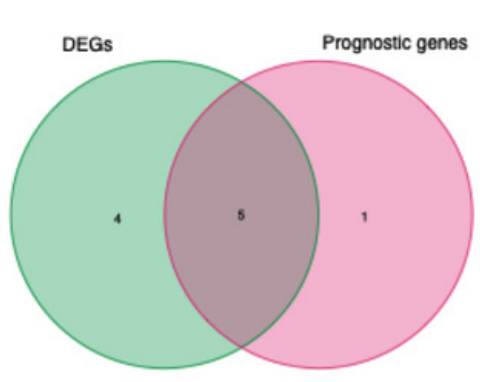

B

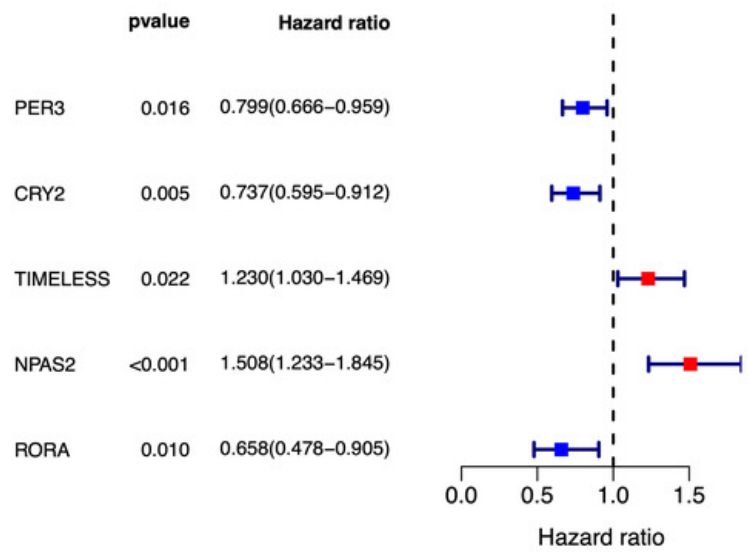

C

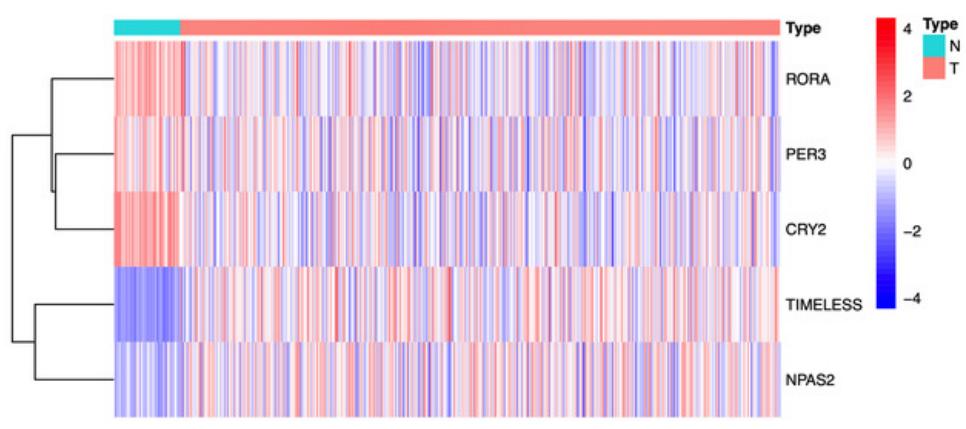

E

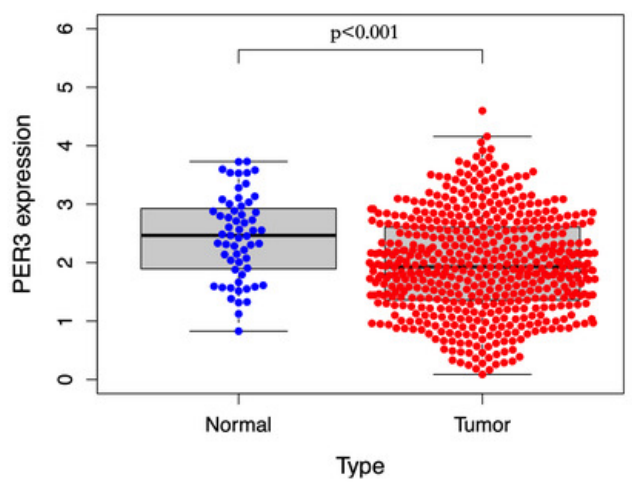

G

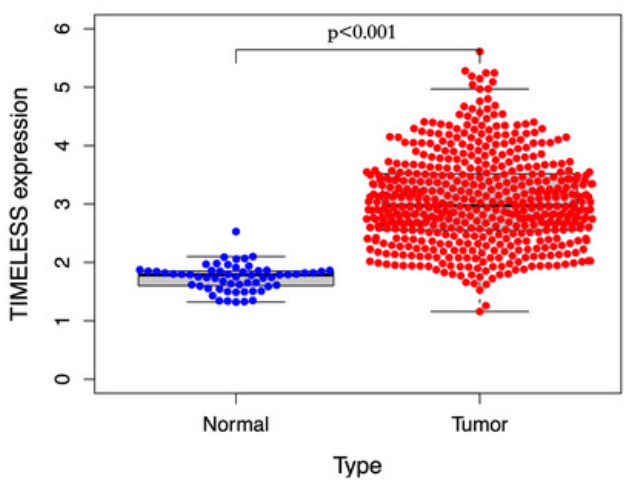

D

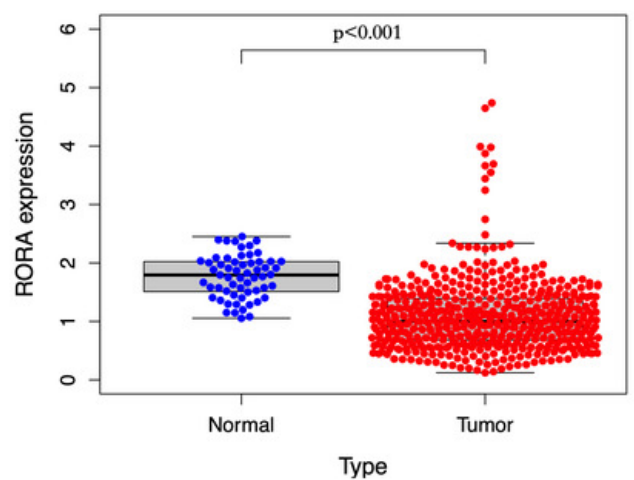

$\mathbf{F}$

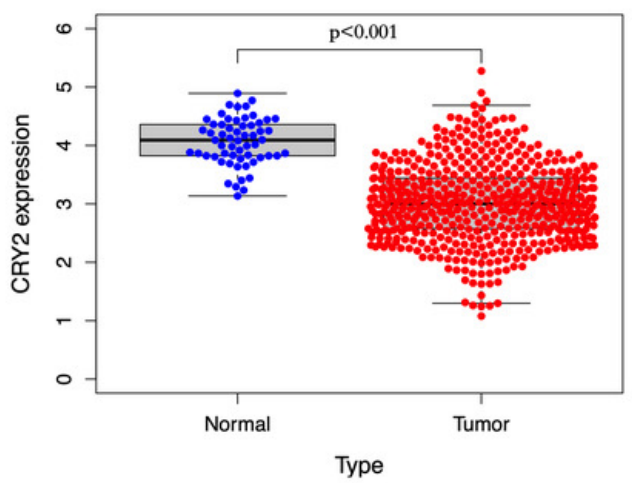

H

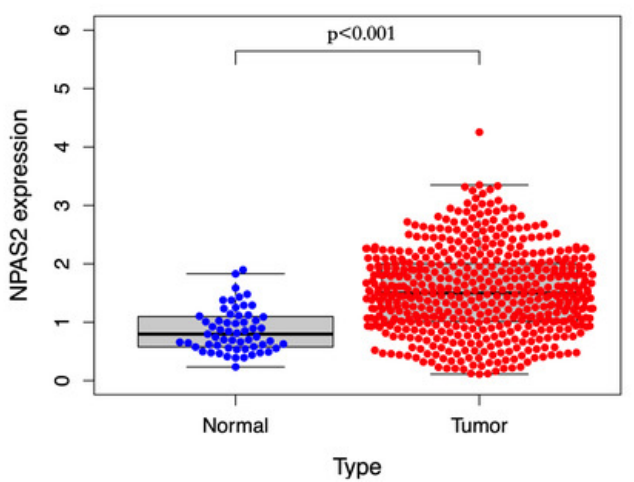


Figure 2

Figure 2. Analysis of the prognostic value of the five-gene-signature based risk score in the TCGA cohort.

(A) The distribution of the risk scores in the TCGA cohort. (B) The distributions of OS status, OS time and risk score in the TCGA cohort. (C) PCA plot of the TCGA cohort. (D) t-SNE analysis of the TCGA cohort. (E) Kaplan-Meier curves for the OS of patients in the highrespective low-risk group in the TCGA cohort. (F) AUC of time-dependent ROC curves that confirm the prognostic performance of the risk score in the TCGA cohort.

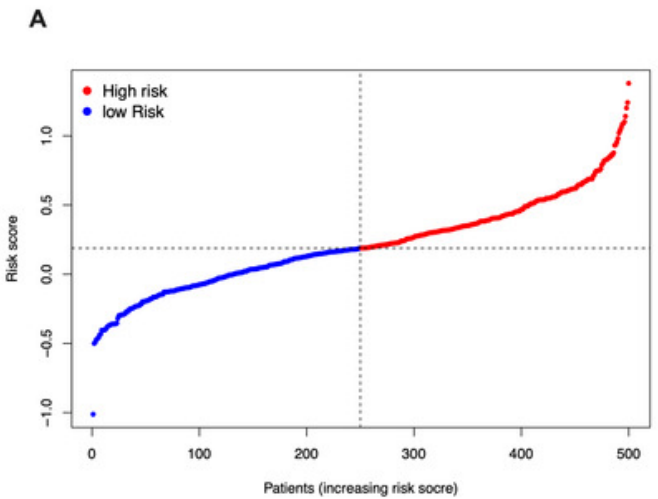

C

D

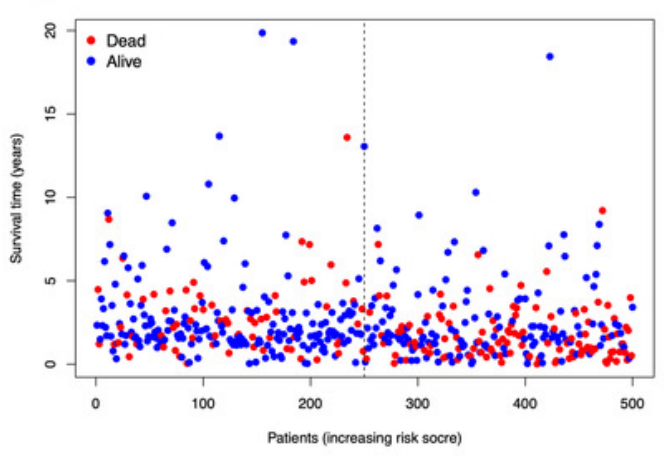

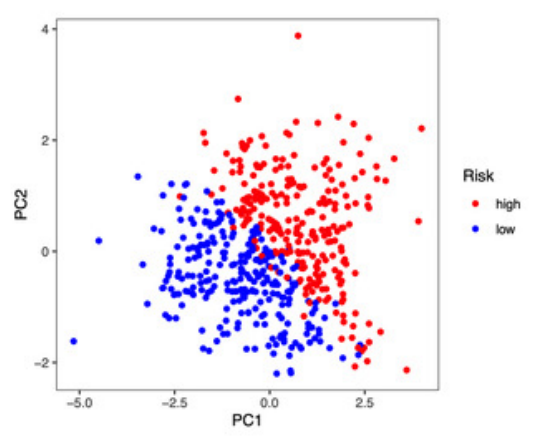

E

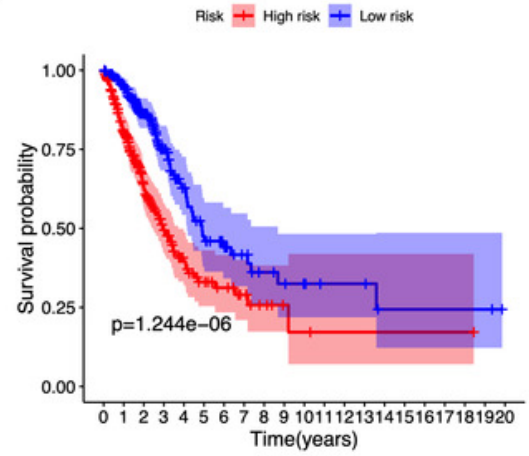

F
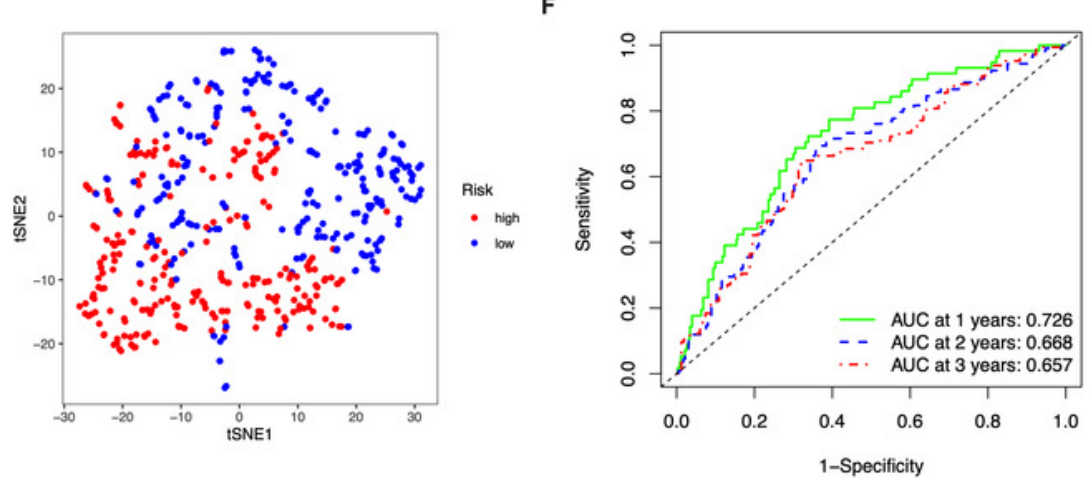


\section{Figure 3}

Figure 3. Validation of the five-gene-signature based risk score in the GSE72094 dataset.

(A) The distribution of the risk scores in the GSE72094 dataset. (B) The distributions of OS status, OS time and risk score in the GSE72094 dataset. (C) PCA plot of the GSE72094 dataset. (D) t-SNE analysis of the GSE72094 dataset. (E) Kaplan-Meier curves for the OS of patients in the high- respective low-risk group in the GSE72094 dataset. (F) AUC of timedependent ROC curves that confirm the prognostic performance of the risk score in the GSE72094 dataset.
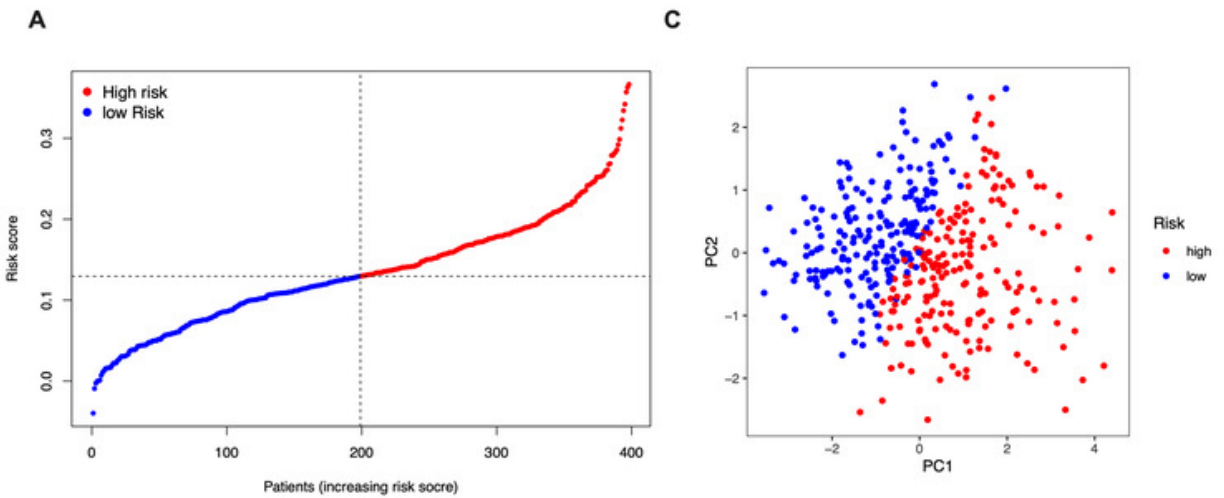

E

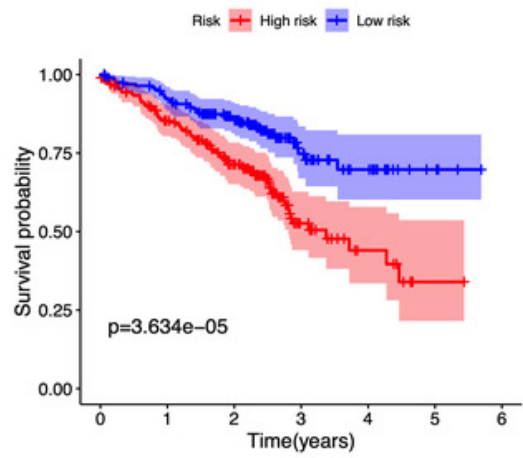

B

D
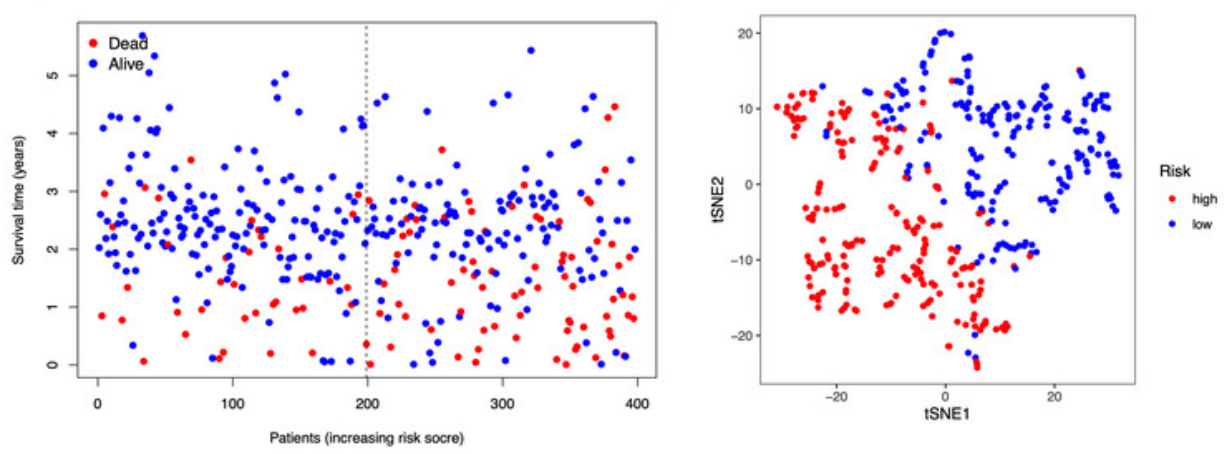

F

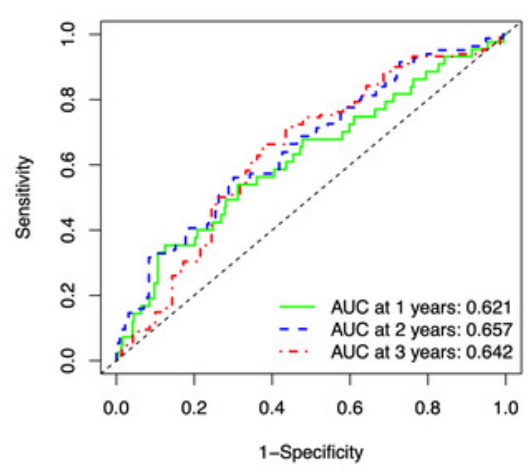


Figure 4

Figure 4. Multivariate Cox regression analyses of factors affecting OS in the TCGA LUAD cohort (A) and the GSE72094 dataset (B).

A

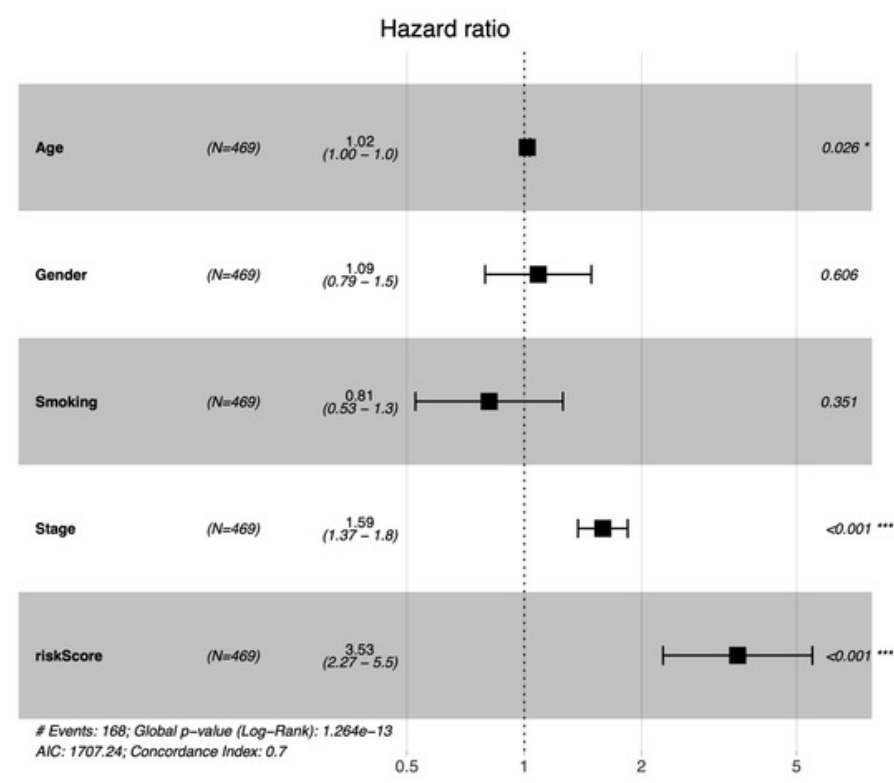

B

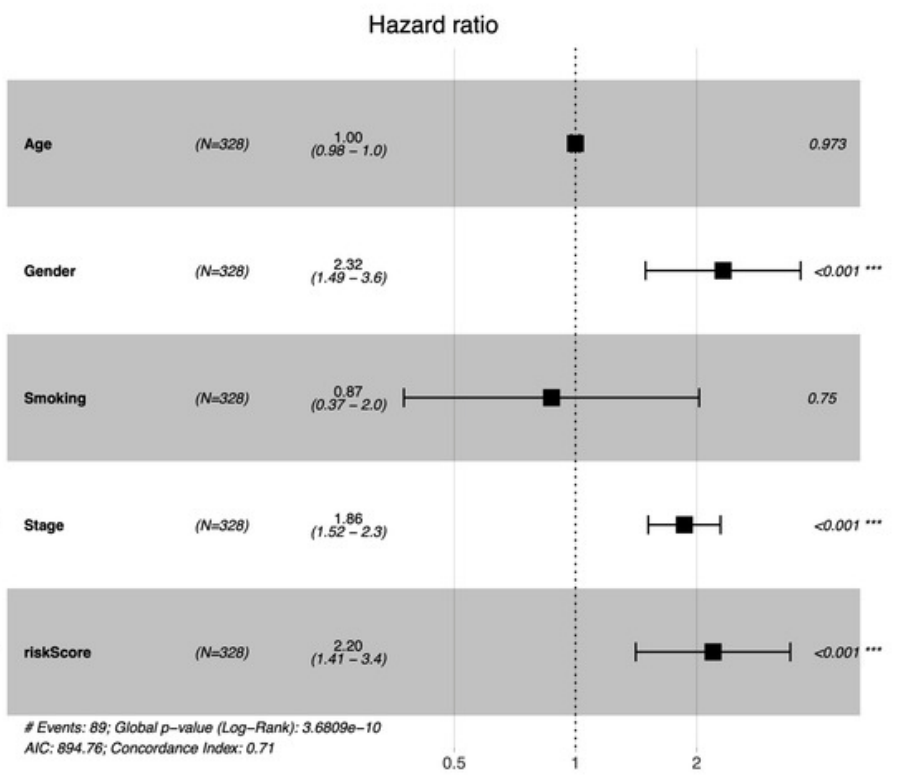


Figure 5

Figure 5. The KEGG enrichment plots of tumorigenesis pathways.

(A) Pyrimidine metabolism. (B) Cell cycle. (C) Proteasome. (D) Base excision repair. (E) Homologous recombination. (F) DNA replication.

A

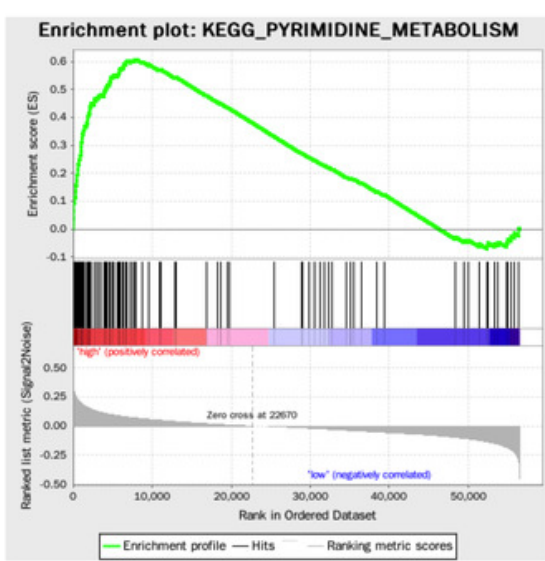

B

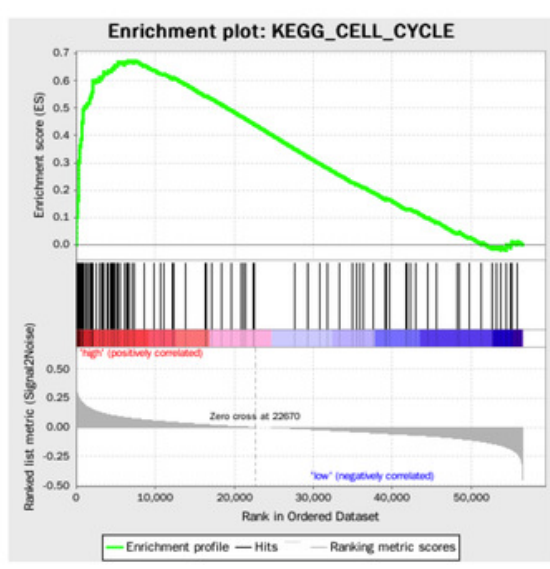

C

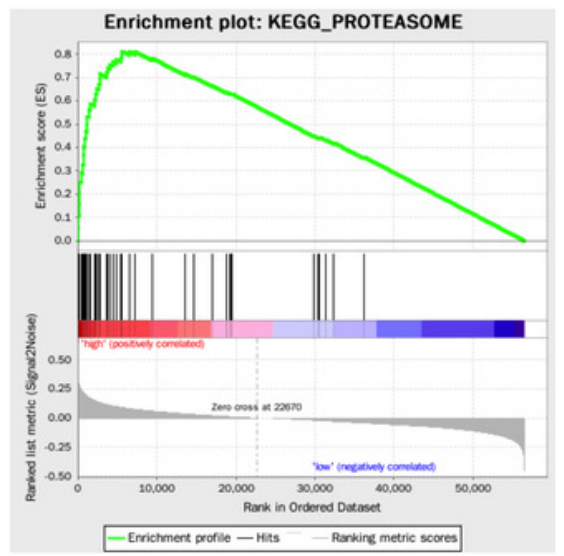

D

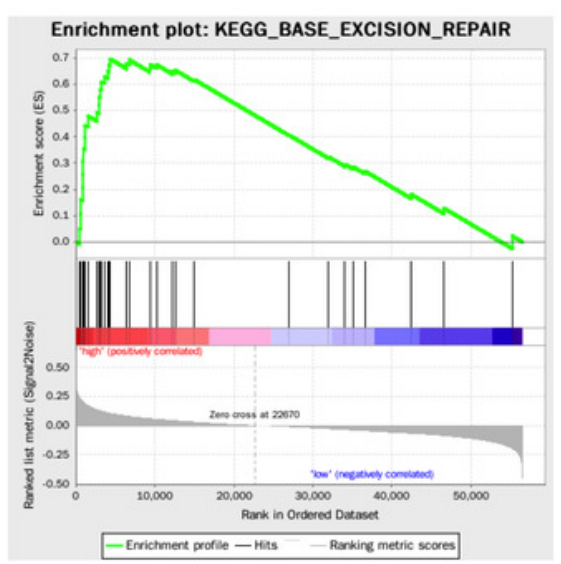

E

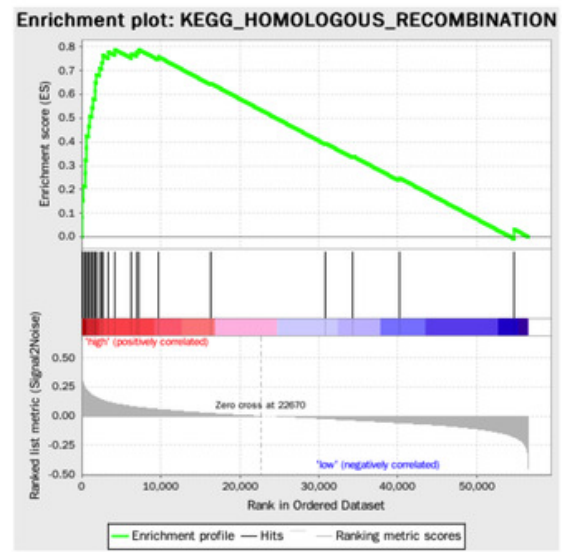

$\mathbf{F}$

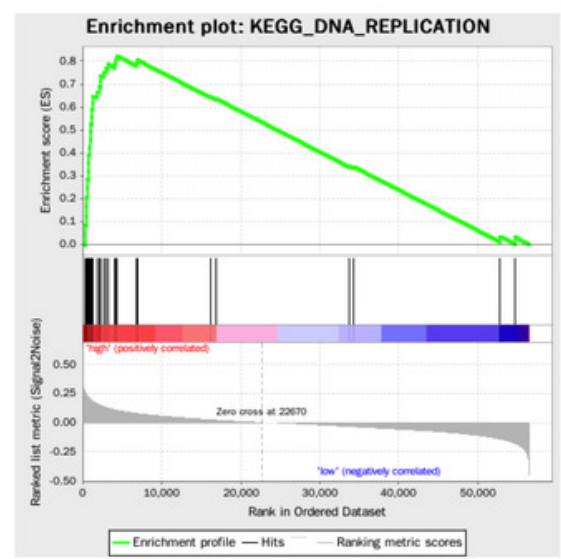

